\title{
Using Parametric Functions to Solve Systems of Linear Fuzzy Equations with a Symmetric Matrix
}

\author{
Annelies Vroman ${ }^{1}$ Glad Deschrijver $^{1}$ Etienne E. Kerre $^{1}$ \\ ${ }^{1}$ Department of Applied Mathematics and Computer Science, Ghent University, Fuzziness and \\ Uncertainty Modelling Research Unit, Krijgslaan 281 (S9), B-9000 Gent, Belgium.
}

\begin{abstract}
A method to solve linear fuzzy equations with a symmetric matrix is proposed. Ignoring the symmetry leads to an overestimation of the solution. Our method to find the solution of a system of linear fuzzy equations takes the symmetry of the matrix into account and is based on parametric functions. It is a practical algorithm using parametric functions in which the variables are given by elements of the support of the fuzzy coefficients of the system.
\end{abstract}

Keywords: Systems of linear fuzzy equations, Symmetry, Parametric functions

\section{Introduction}

In this paper we search for a solution of the matrix equation: $\tilde{A}_{s y m} \tilde{\mathbf{x}}=\tilde{\mathbf{b}}$ for $\tilde{\mathbf{x}}=\left[\tilde{x}_{k}\right]_{n \times 1}$ where $\tilde{A}_{s y m}=\left[\tilde{a}_{i j}\right]_{n \times n}$ is a symmetric matrix with fuzzy numbers as entries and $\tilde{\mathbf{b}}=\left[\tilde{b}_{k}\right]_{n \times 1}$ is a vector of fuzzy numbers. Differently expressed,

$$
\sum_{j=1}^{n} \tilde{a}_{i j} \tilde{x}_{j}=\tilde{b}_{i}, \quad \text { for } 1 \leq i \leq n,
$$

where fuzzy multiplication and addition based on the extension principle of Zadeh are used. Taking the $\alpha$-levels of these equations we obtain systems of linear interval equations:

$$
\sum_{j=1}^{n}\left[\left(\underline{a}_{i j}\right)_{\alpha},\left(\bar{a}_{i j}\right)_{\alpha}\right]\left[\left(\underline{x}_{j}\right)_{\alpha},\left(\bar{x}_{j}\right)_{\alpha}\right]=\left[\left(\underline{b}_{i}\right)_{\alpha},\left(\bar{b}_{i}\right)_{\alpha}\right],
$$

for $0<\alpha \leq 1$ and $1 \leq i \leq n$. The exact solution is then given by

$\left(\tilde{\mathbf{x}}_{e}\right)_{i}(x)=\sup \left\{\alpha \mid \alpha \in[0,1]\right.$ and $\left.x \in\left[\left(\underline{x}_{i}\right)_{\alpha},\left(\bar{x}_{i}\right)_{\alpha}\right]\right\}$, for all $x \in \mathbb{R}$.
When this solution is entered into the system the equations are satisfied. However, these interval equations are hard to solve exactly and often $\left(\underline{x}_{j}\right)_{\alpha}$ and $\left(\bar{x}_{j}\right)_{\alpha}$ do not generate a fuzzy number (see [1]). Therefore the search for an alternative solution has a solid ground. Buckley and Qu [1] have already proposed a solution. We follow their line of reasoning, although the solution can be adjusted a little bit and we consider the symmetry of the matrix. A practical algorithm to obtain this solution, where we take the symmetry into account, is proposed here. The original method based on parametric functions for solving systems of linear fuzzy equations with non-symmetric matrices is described in [4] and [5].

\section{Preliminaries}

First we recall some definitions concerning fuzzy numbers [3]. Let $A$ be a fuzzy set on $\mathbb{R}$. Then $A$ is called convex if

$$
A\left(\lambda x_{1}+(1-\lambda) x_{2}\right) \geq \min \left(A\left(x_{1}\right), A\left(x_{2}\right)\right),
$$

for all $x_{1}, x_{2} \in \mathbb{R}$ and $\lambda \in[0,1]$. If for $x \in \mathbb{R}$ it holds that $A(x)=1$, then we call $x$ a modal value of $A$. The support of $A$ is defined as

$$
\operatorname{supp} A=\{x \mid x \in \mathbb{R} \text { and } A(x)>0\} .
$$

For all $\alpha \in[0,1]$, the $\alpha$-level is defined as the set:

$$
A_{\alpha}= \begin{cases}\{x \mid x \in \mathbb{R} \text { and } A(x) \geq \alpha\} & \text { if } \alpha>0, \\ \overline{\{x \mid x \in \mathbb{R} \text { and } A(x)>0\}} & \text { if } \alpha=0 .\end{cases}
$$

A mapping $f: \mathbb{R} \rightarrow \mathbb{R}$, or in particular $f$ : $\mathbb{R} \rightarrow[0,1]$, is called upper-semicontinuous when $f$ is right-continuous where $f$ is increasing, and leftcontinuous where $f$ is decreasing.

Definition 1 [3]. A fuzzy number is defined as a convex upper-semicontinuous fuzzy set on $\mathbb{R}$ with a unique modal value and bounded support. 
From now on fuzzy numbers will be denoted by a lowercase letter with a tilde, e.g. $\tilde{a}$, and a vector of fuzzy numbers will be denoted as $\tilde{\mathbf{b}}$. Sometimes we will denote the $i$-th component of $\tilde{\mathbf{b}}$ by $\tilde{b}_{i}$. Crisp numbers will be represented by a lowercase letter, e.g. $a$, and vectors of crisp numbers will be denoted as $\mathbf{b}=\left(b_{1}, b_{2}, \ldots, b_{n}\right)^{T}$. The notions of support and $\alpha$-level are extended componentwise for vectors or matrices of fuzzy numbers. The arithmetic of fuzzy numbers is based on Zadeh's extension principle. Let $\tilde{a}$ and $\tilde{b}$ be two fuzzy numbers, then the sum of $\tilde{a}$ and $\tilde{b}$, denoted by $\tilde{a} \oplus \tilde{b}$, is given by, for all $z \in \mathbb{R}$,

$$
(\tilde{a} \oplus \tilde{b})(z)=\sup _{z=x+y} \min (\tilde{a}(x), \tilde{b}(y)) .
$$

Analogous definitions follow for the fuzzy multiplication, subtraction and division. The fuzzy arithmetic based on the sup-min convolution (see (1)) can also be calculated by interval arithmetic applied to the $\alpha$-levels.

Definition $2[2]$. Given two intervals $[\underline{x}, \bar{x}] \subseteq \mathbb{R}$ and $[y, \bar{y}] \subseteq \mathbb{R}$, the four elementary operations on intervals are defined by

$[\underline{x}, \bar{x}]$ op $[\underline{y}, \bar{y}]=\{x$ op $y \mid x \in[\underline{x}, \bar{x}]$ and $y \in[\underline{y}, \bar{y}]\}$, for op $\in\{+, \times,-, \div\}$.

It is well-known that $(\tilde{a} \oplus \tilde{b})_{\alpha}=\tilde{a}_{\alpha}+\tilde{b}_{\alpha}$ and similarly for $\otimes$.

\section{Solving systems of linear fuzzy equations}

First of all, we require that the matrix $\tilde{A}_{\text {sym }}$ of fuzzy numbers is regular in the sense that the inverse matrix of $A$ exists for all $a_{i j} \in \operatorname{supp}\left(\tilde{a}_{i j}\right)$ with $a_{i j}=a_{j i}$ for all $(i, j) \in\{1, \ldots, n\}^{2}$.

Buckley and $\mathrm{Qu}[1]$ proposed to construct a set of all crisp solutions corresponding to the crisp systems formed by the elements in a certain $\alpha$-level. They define the solution by, for all $\alpha \in[0,1]$,

$$
\begin{array}{r}
\Omega(\alpha)=\left\{\mathbf{x} \mid \mathbf{x} \in \mathbb{R}^{n} \text { and }\left(\exists A=\left[a_{i j}\right]_{n \times n} \in \mathbb{R}^{n \times n}\right)\right. \\
\left(\exists \mathbf{b}=\left[b_{k}\right]_{n \times 1} \in \mathbb{R}^{n}\right)\left(\left(\forall(i, j, k) \in\{1,2, \ldots, n\}^{3}\right)\right. \\
\left.\left.\left(a_{i j} \in\left(\tilde{a}_{i j}\right)_{\alpha} \text { and } b_{k} \in\left(\tilde{b}_{k}\right)_{\alpha}\right) \text { and } A \mathbf{x}=\mathbf{b}\right)\right\}
\end{array}
$$

and for all $\mathbf{x} \in \mathbb{R}^{n}$,

$$
\tilde{\mathbf{x}}_{B}(\mathbf{x})=\sup \{\alpha \mid \alpha \in[0,1] \text { and } \mathbf{x} \in \Omega(\alpha)\} .
$$

We see that $\tilde{\mathbf{x}}_{B}$ is defined as a fuzzy set on $\mathbb{R}^{n}$ and not as a vector of fuzzy numbers. The solution $\tilde{\mathbf{x}}_{B}(\mathbf{x})$ expresses to what extent the crisp vector $\mathbf{x}$ is a solution of the system of linear fuzzy equations $\tilde{A} \tilde{\mathbf{x}}=\tilde{\mathbf{b}}$. We prefer to define the solution as a vector of fuzzy numbers to avoid information loss. Therefore we give a membership degree to every component of the solution vector and then $\left(\tilde{\mathbf{x}}_{B}\right)_{i}(x)$ expresses the degree to which $x$ belongs to the fuzzy set $\left(\tilde{\mathbf{x}}_{B}\right)_{i}$, independent of $\left(\tilde{\mathbf{x}}_{B}\right)_{j}$, for all $j \neq i$. We thus define for all $x \in \mathbb{R}$ and for all $i \in\{1,2, \ldots, n\}$

$$
\begin{aligned}
\left(\tilde{\mathbf{x}}_{B}\right)_{i}(x)= & \sup \{\alpha \mid \alpha \in[0,1] \text { and } \\
& \left.(\exists \mathbf{x} \in \Omega(\alpha))\left(x=x_{i}\right)\right\},
\end{aligned}
$$

where $x_{i}$ denotes the $i$-th component of $\mathbf{x}$. This method is purely theoretical: in fact all crisp systems are solved. When all these systems have to be solved, the computation time will be large. An other drawback of this method proposed by Buckley and $\mathrm{Qu}$ is that the symmetry isn't taken into account. In this paper we propose a practical algorithm to compute the solution where the symmetry is also taken into account. Instead of solving all these crisp systems with a symmetric matrix, we determine parametric functions with elements of the support of the fuzzy numbers as variables of these solutions.

\subsection{Systems with one fuzzy co- efficient}

We first consider the case that we have to solve a system of linear fuzzy equations in which exactly one of the coefficients is a fuzzy number and the other coefficients are crisp. The approach is different for a fuzzy non-diagonal or a fuzzy diagonal element of the matrix or a fuzzy component of the vector $\tilde{\mathbf{b}}$.

\subsubsection{The fuzzy coefficient is a diagonal element of the matrix $\tilde{A}$}

First of all we consider that the fuzzy number is a diagonal element of the matrix. Without loss of generality we may assume that $\tilde{a}_{11}$ is a fuzzy number. In order to obtain the solution $\tilde{\mathbf{x}}_{\text {sym }}$ of $\tilde{A}_{\text {sym }} \tilde{\mathbf{x}}=\tilde{\mathbf{b}}$, we have to solve the crisp systems $A^{s}\left(a_{11}\right) \mathbf{x}=\mathbf{b}$, where for all $\left.a_{11} \in\right] \underline{a}_{11}, \bar{a}_{11}[=$ $\operatorname{supp}\left(\tilde{a}_{11}\right)$ where

$$
A^{s}\left(a_{11}\right)=\left(\begin{array}{cccc}
a_{11} & a_{12} & \cdots & a_{1 n} \\
a_{12} & a_{22} & \cdots & a_{2 n} \\
\vdots & \vdots & \ddots & \vdots \\
a_{1 n} & a_{2 n} & \cdots & a_{n n}
\end{array}\right),
$$




$$
\begin{aligned}
& \mathbf{x}=\left(\begin{array}{c}
x_{1} \\
x_{2} \\
\vdots \\
x_{n}
\end{array}\right), \\
& \mathbf{b}=\left(\begin{array}{c}
b_{1} \\
b_{2} \\
\vdots \\
b_{n}
\end{array}\right),
\end{aligned}
$$

where $A^{s}\left(a_{11}\right)$ is obtained by replacing $\tilde{a}_{11}$ with $a_{11}$ in the matrix $\tilde{A}_{\text {sym }}$. We can solve all of these systems through Cramer's rule thanks to the nonsingularity of the crisp matrix $A^{s}\left(a_{11}\right)$, for all $a_{11} \in$ $\operatorname{supp}\left(\tilde{a}_{11}\right)$. So we can write the solution for every component as a quotient of two determinants. The determinant of a matrix $A$ is denoted as $|A|$.

$$
x_{j}=\frac{\left|\begin{array}{ccccc}
j & & \\
a_{11} & \cdots & b_{1} & \cdots & a_{1 n} \\
\vdots & \ddots & \vdots & \ddots & \vdots \\
a_{1 n} & \cdots & b_{n} & \cdots & a_{n n}
\end{array}\right|}{\left|\begin{array}{cccc}
a_{11} & a_{12} & \cdots & a_{1 n} \\
a_{12} & a_{22} & \cdots & a_{2 n} \\
\vdots & \vdots & \ddots & \vdots \\
a_{1 n} & a_{2 n} & \cdots & a_{n n}
\end{array}\right|} .
$$

By expanding the determinants in the numerator and the denominator along the first row, we can write each component of the solution using parameters $c_{1 j}, c_{2 j}, c_{3}$ and $c_{4}$ :

$$
x_{j}=f_{j}\left(a_{11}\right)=\frac{a_{11} c_{1 j}+c_{2 j}}{a_{11} c_{3}+c_{4}} .
$$

Due to this result, every solution can be written using parametric functions with variable $a_{11}$. Note that $c_{1 j}$ and $c_{2 j}$ are dependent of $j$ due to the fact that the $j$-th column in the numerator contains the components of $\mathbf{b}$. On the other hand, the denominator is the same for all $j \in\{1, \ldots, n\}$, so $c_{3}$ and $c_{4}$ are independent of $j$. Thus we propose the following method to solve $\tilde{A}_{\text {sym }} \tilde{\mathbf{x}}=\tilde{\mathbf{b}}$. First we compute the determinant of the matrices $A^{s}\left(\underline{a}_{11}\right)$ and $A^{s}\left(\bar{a}_{11}\right)$. The parameters $c_{3}$ and $c_{4}$ are obtained by solving the following system of linear crisp equations:

$$
\left\{\begin{array}{l}
\left|A^{s}\left(\underline{a}_{11}\right)\right|=\underline{a}_{11} c_{3}+c_{4} \\
\left|A^{s}\left(\bar{a}_{11}\right)\right|=\bar{a}_{11} c_{3}+c_{4} .
\end{array}\right.
$$

We solve the crisp systems

$$
\begin{aligned}
& A^{s}\left(\underline{a}_{11}\right) \mathbf{x}=\mathbf{b}, \\
& A^{s}\left(\bar{a}_{11}\right) \mathbf{x}=\mathbf{b},
\end{aligned}
$$

and denote by

$$
\underline{\mathbf{x}}=\left(\underline{x}_{1}, \ldots, \underline{x}_{n}\right)^{T}
$$

and

$$
\overline{\mathbf{x}}=\left(\bar{x}_{1}, \ldots, \bar{x}_{n}\right)^{T}
$$

the solutions of (4) and (5) respectively. Then, for all $j \in\{1, \ldots, n\}$, we obtain $c_{1 j}$ and $c_{2 j}$ by solving the following system of crisp equations:

$$
\left\{\begin{array}{l}
\underline{x}_{j}\left|A^{s}\left(\underline{a}_{11}\right)\right|=\underline{a}_{11} c_{1 j}+c_{2 j} \\
\bar{x}_{j}\left|A^{s}\left(\bar{a}_{11}\right)\right|=\bar{a}_{11} c_{1 j}+c_{2 j} .
\end{array}\right.
$$

Consequently, all possible solutions for the crisp systems $A^{s}\left(a_{11}\right) \mathbf{x}=\mathbf{b}$, for all $a_{11} \in \operatorname{supp}\left(\tilde{a}_{11}\right)$, can be obtained using (2). We define for all $j \in$ $\{1, \ldots, n\}$ the fuzzy number $\tilde{x}_{j}^{\text {sym }}$ as:

$$
\begin{array}{r}
\tilde{x}_{j}^{s y m}(x)=\sup \left\{\tilde{a}_{11}\left(a_{11}\right) \mid a_{11} \in \operatorname{supp}\left(\tilde{a}_{11}\right)\right. \\
\text { and } \left.x=f_{j}\left(a_{11}\right)\right\},
\end{array}
$$

for all $x \in f_{j}\left(\operatorname{supp}\left(\tilde{a}_{11}\right)\right)$, and

$$
\tilde{x}_{j}^{s y m}(x)=0,
$$

for all $x \in \mathbb{R} \backslash f_{j}\left(\operatorname{supp}\left(\tilde{a}_{11}\right)\right)$.

\subsubsection{The fuzzy coefficient is a component of the vector $\tilde{\mathbf{b}}$}

When the fuzzy number is located in the right-hand side of the system of linear fuzzy equations, i.e. , when we have for instance that $\tilde{\mathbf{b}}=\left(\tilde{b}_{1}, b_{2}, \ldots, b_{n}\right)$, one sees immediately that $c_{3}=0$ and $c_{4}=|A|$. So we only have to solve the systems

$$
\begin{aligned}
& A \mathbf{x}=\mathbf{b}\left(\underline{b}_{1}\right), \\
& A \mathbf{x}=\mathbf{b}\left(\bar{b}_{1}\right),
\end{aligned}
$$

with $\mathbf{b}\left(\underline{b}_{1}\right)$ and $\mathbf{b}\left(\bar{b}_{1}\right)$ a crisp vector obtained by replacing the fuzzy number $\tilde{b}_{1}$ by the lower and upper bound resp. of its support, and

$$
\left\{\begin{array}{l}
\underline{x}_{j}|A|=\underline{b}_{1} c_{1 j}+c_{2 j} \\
\bar{x}_{j}|A|=\bar{b}_{1} c_{1 j}+c_{2 j}
\end{array}\right.
$$

to find $c_{1 j}$ and $c_{2 j}$ for $j \in\{1, \ldots, n\}$. The function $f_{j}$ is then given by, for all $j \in\{1, \ldots, n\}$,

$$
f_{j}\left(b_{1}\right)=\frac{b_{1} c_{1 j}+c_{2 j}}{|A|},
$$

and the solution $\tilde{\mathbf{x}}_{\text {sym }}=\left(\tilde{x}_{1}, \ldots, \tilde{x}_{n}\right)^{T}$ is given by, for all $j \in\{1, \ldots, n\}$,

$\tilde{x}_{j}\left(x_{j}\right)=\sup \left\{\tilde{b}_{1}\left(b_{1}\right) \mid b_{1} \in \operatorname{supp}\left(\tilde{b}_{1}\right)\right.$ and $\left.x_{j}=f_{j}\left(b_{1}\right)\right\}$, for all $x_{j} \in f\left(\operatorname{supp}\left(\tilde{b}_{1}\right)\right)$, and

$$
\tilde{x}_{j}\left(x_{j}\right)=0,
$$

for all $x_{j} \in \mathbb{R} \backslash f\left(\operatorname{supp}\left(\tilde{b}_{1}\right)\right)$. 


\subsubsection{The fuzzy coefficient is a non-diagonal ele- ment of the matrix $\tilde{A}$}

Now we assume that the fuzzy coefficient is a nondiagonal element of the matrix $\tilde{A}_{\text {sym }}$. Without loss of generality, we may assume that the element on the second column and the first row and consequently on the first column and the second row is the fuzzy number in $\tilde{A}$ :

$$
\left(\begin{array}{cccc}
a_{11} & \tilde{a}_{12} & \cdots & a_{1 n} \\
\tilde{a}_{12} & a_{22} & \cdots & a_{2 n} \\
\vdots & \vdots & \ddots & \vdots \\
a_{1 n} & a_{2 n} & \cdots & a_{n n}
\end{array}\right)\left(\begin{array}{c}
\tilde{x}_{1} \\
\tilde{x}_{2} \\
\vdots \\
\tilde{x}_{n}
\end{array}\right)=\left(\begin{array}{c}
b_{1} \\
b_{2} \\
\vdots \\
b_{n}
\end{array}\right) .
$$

To find the solution considering the symmetry of the matrix, we have to solve

$$
A^{s}\left(a_{12}\right) \mathbf{x}=\mathbf{b},
$$

where

$$
A^{s}\left(a_{12}\right)=\left(\begin{array}{cccc}
a_{11} & a_{12} & \cdots & a_{1 n} \\
a_{12} & a_{22} & \cdots & a_{2 n} \\
\vdots & \vdots & \ddots & \vdots \\
a_{1 n} & a_{2 n} & \cdots & a_{n n}
\end{array}\right)
$$

for all $\left.a_{12} \in\right] \underline{a}_{12}, \bar{a}_{12}\left[=\operatorname{supp}\left(\tilde{a}_{12}\right)\right.$.

Analogous to the previous case, all these crisp systems can be solved by Cramer's rule because each real symmetric matrix $A^{s}\left(a_{12}\right)$ for all $a_{12} \in$ $\operatorname{supp}\left(\tilde{a}_{12}\right)$ is regular. So we can write each component as the quotient of two determinants:

$$
x_{j}=\frac{\left|\begin{array}{ccccc} 
& j & & \\
a_{11} & \cdots & b_{1} & \cdots & a_{1 n} \\
\vdots & \ddots & \vdots & \ddots & \vdots \\
a_{1 n} & \cdots & b_{n} & \cdots & a_{n n}
\end{array}\right|}{\left|\begin{array}{cccc}
a_{11} & a_{12} & \cdots & a_{1 n} \\
a_{12} & a_{22} & \cdots & a_{2 n} \\
\vdots & \vdots & \ddots & \vdots \\
a_{1 n} & a_{2 n} & \cdots & a_{n n}
\end{array}\right|} .
$$

By expanding these determinants along the first row, each component of the solution can be written using 6 parameters $c_{1 j}, c_{2 j}, c_{3 j}, c_{4}, c_{5}$ and $c_{6}$ :

$$
x_{j}=f_{j}^{s y m}\left(a_{12}\right)=\frac{a_{12}^{2} c_{1 j}+a_{12} c_{2 j}+c_{3 j}}{a_{12}^{2} c_{4}+a_{12} c_{5}+c_{6}} .
$$

Each solution of $A^{s}\left(a_{12}\right) \mathbf{x}=\mathbf{b}$ can be expressed using the parametric functions with $a_{12}$ as variable. First the determinants of the matrices $A^{s}\left(\underline{a}_{12}\right)$,
$A^{s}\left(\left(\tilde{a}_{12}\right)_{1}\right)$ and $A^{s}\left(\bar{a}_{12}\right)$ are calculated. The values of the parameters $c_{4}, c_{5}$ and $c_{6}$ can be found through the following system of linear equations:

$$
\left\{\begin{aligned}
\left|A^{s}\left(\underline{a}_{12}\right)\right| & =\underline{a}_{12}^{2} c_{4}+\underline{a}_{12} c_{5}+c_{6} \\
\left|A^{s}\left(\left(\tilde{a}_{12}\right)_{1}\right)\right| & =\left(\tilde{a}_{12}\right)_{1}^{2} c_{4}+\left(\tilde{a}_{12}\right)_{1} c_{5}+c_{6} \\
\left|A^{s}\left(\bar{a}_{12}\right)\right| & =\bar{a}_{12}^{2} c_{4}+\bar{a}_{12} c_{5}+c_{6} .
\end{aligned}\right.
$$

Thereafter the crisp systems:

$$
\begin{aligned}
A^{s}\left(\underline{a}_{12}\right) \mathbf{x} & =\mathbf{b}, \\
A^{s}\left(\left(\tilde{a}_{12}\right)_{1}\right) \mathbf{x} & =\mathbf{b}, \\
A^{s}\left(\bar{a}_{12}\right) \mathbf{x} & =\mathbf{b},
\end{aligned}
$$

are solved with for example Gaussian elimination. The solutions of (9), (10) and (11) are denoted as

$$
\begin{gathered}
\underline{\mathbf{x}}=\left(\underline{x}_{1}, \ldots, \underline{x}_{n}\right)^{T}, \\
(\mathbf{x})_{1}=\left(\left(x_{1}\right)_{1}, \ldots,\left(x_{n}\right)_{1}\right)^{T}
\end{gathered}
$$

and

$$
\overline{\mathbf{x}}=\left(\bar{x}_{1}, \ldots, \bar{x}_{n}\right)^{T}
$$

respectively. The values of the parameters $c_{1 j}, c_{2 j}$ and $c_{3 j}$ are obtained as the solution of the following system of linear equations:

$$
\left\{\begin{aligned}
\underline{x}_{j}\left|A^{s}\left(\underline{a}_{12}\right)\right| & =\underline{a}_{12}^{2} c_{1 j}+\underline{a}_{12} c_{2 j}+c_{3 j} \\
\left(x_{j}\right)_{1}\left|A^{s}\left(\left(\tilde{a}_{12}\right)_{1}\right)\right| & =\left(\tilde{a}_{12}\right)_{1}^{2} c_{1 j}+\left(\tilde{a}_{12}\right)_{1} c_{2 j}+c_{3 j} \\
\bar{x}_{j}\left|A^{s}\left(\bar{a}_{12}\right)\right| & =\bar{a}_{12}^{2} c_{1 j}+\bar{a}_{12} c_{2 j}+c_{3 j} .
\end{aligned}\right.
$$

In that way all the possible solutions of the linear systems $A^{s}\left(a_{12}\right) \mathbf{x}=\mathbf{b}$, for all $a_{12} \in \operatorname{supp}\left(\tilde{a}_{12}\right)$ are calculated by (8). We define for all $j \in$ $\{1, \ldots, n\}$ the fuzzy number $\tilde{x}_{j}^{\text {sym }}$ as:

$$
\begin{array}{r}
\tilde{x}_{j}^{s y m}(x)=\sup \left\{\tilde{a}_{12}\left(a_{12}\right) \mid a_{12} \in \operatorname{supp}\left(\tilde{a}_{12}\right)\right. \\
\text { and } \left.x=f_{j}^{s y m}\left(a_{12}\right)\right\},
\end{array}
$$

for all $x \in f_{j}^{\text {sym }}\left(\operatorname{supp}\left(\tilde{a}_{12}\right)\right)$, and

$$
\tilde{x}_{j}^{s y m}(x)=0,
$$

for all $x \in \mathbb{R} \backslash f_{j}^{\text {sym }}\left(\operatorname{supp}\left(\tilde{a}_{12}\right)\right)$.

\subsection{Systems with two fuzzy co- efficients}

In this section we consider a system of two fuzzy coefficients $\widetilde{F n_{1}}$ and $\widetilde{F n_{2}}$. The approach differs for a fuzzy diagonal or fuzzy non-diagonal element of the matrix $\tilde{A}$ or a fuzzy component of the vector $\tilde{\mathbf{b}}$. 


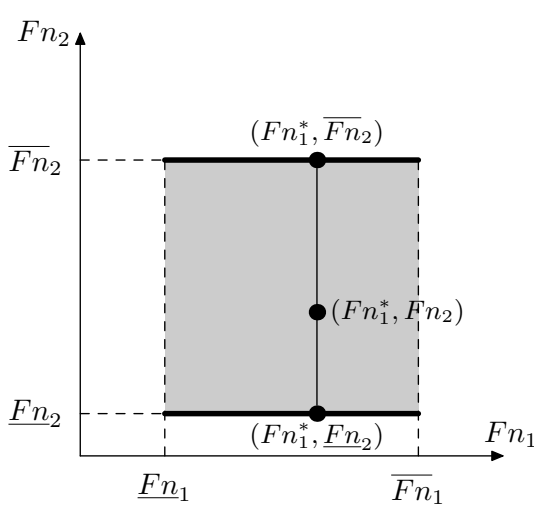

Fig. 1: Solving systems with two fuzzy coefficients.

\subsubsection{The second fuzzy coefficient is a diagonal element of the matrix $\tilde{A}$}

Assume the second fuzzy number is a diagonal element of the matrix $\tilde{A}$. We first fix the second fuzzy number on the lower bound of its support. In that way we can find the solutions for the systems corresponding to the lower line of the rectangle in Figure 1 by the method described in Section 3.1. Analogously we fix the second fuzzy number on the upper bound of its support and find the solutions for the upper line in Figure 1. Thereafter we fix arbitrarily the first fuzzy number on $F n_{1}^{*} \in \operatorname{supp}\left(\widetilde{F n_{1}}\right)$ and let $F n_{2} \in \operatorname{supp}\left(\widetilde{F n_{2}}\right)$ vary. So, again, we obtain a system with only one fuzzy number, but this time the fuzzy number is $\widetilde{\mathrm{Fn}_{2}}$. Thus we are looking for the solution of the crisp systems corresponding to the points on the vertical thin line in Figure 1. Similarly as we did before, we can obtain the solution of the crisp system $A^{s}\left(F n_{1}^{*}, F n_{2}\right) \mathbf{x}=\mathbf{b}$ as

$$
x_{j}=f_{j}^{*}\left(F n_{2}\right)=\frac{F n_{2} c_{1 j}^{*}+c_{2 j}^{*}}{F n_{2} c_{3}^{*}+c_{4}^{*}}
$$

for all $j \in\{1, \ldots, n\}$ and $F n_{2} \in \operatorname{supp}\left(\widetilde{F n_{2}}\right)$. We find the parameters $c_{3}^{*}$ and $c_{4}^{*}$ by solving the system

$$
\left\{\begin{array}{l}
f_{\text {denom }}^{l}\left(F n_{1}^{*}\right)=\underline{F n}_{2} c_{3}^{*}+c_{4}^{*} \\
f_{\text {denom }}^{u}\left(F n_{1}^{*}\right)=\overline{F n}_{2} c_{3}^{*}+c_{4}^{*} .
\end{array}\right.
$$

where $f_{\text {denom }}^{l}\left(F n_{1}^{*}\right)$ and $f_{\text {denom }}^{u}\left(F n_{1}^{*}\right)$ are the denominators of the parametric functions for respectively the lower $(l)$ and the upper $(u)$ bound of the support of $\widetilde{F n_{2}}$, with the first fuzzy number as variable and evaluated in the fixed value $F n_{1}^{*}$. Then, for all $j \in\{1, \ldots, n\}$, we obtain $c_{1 j}^{*}$ and $c_{2 j}^{*}$ by solving the following system:

$$
\left\{\begin{array}{l}
f_{\text {num }, j}^{l}\left(F n_{1}^{*}\right)=\underline{F n_{2}} c_{1 j}^{*}+c_{2 j}^{*} \\
f_{\text {num }, j}^{u}\left(F n_{1}^{*}\right)=\overline{F n}_{2} c_{1 j}^{*}+c_{2 j}^{*} .
\end{array}\right.
$$

where $f_{n u m, j}^{l}\left(F n_{1}^{*}\right)$ and $f_{n u m, j}^{u}\left(F n_{1}^{*}\right)$ are the numerators of the $j$-th component of the parametric functions for respectively the lower $(l)$ and the upper $(u)$ bound of the support of $\widetilde{F n_{2}}$, with the first fuzzy number as variable and evaluated in the fixed value $F n_{1}^{*}$. Consequently, all possible solutions for the crisp systems $A\left(F n_{1}^{*}, F n_{2}\right) \mathbf{x}=\mathbf{b}$, for all $F n_{2} \in \operatorname{supp}\left(\widetilde{F n_{2}}\right)$, can be obtained using (13). This approach can be used independently of the place of the first fuzzy number; it doesn't matter if the first fuzzy number is a diagonal or non-diagonal element of the matrix $\tilde{A}$ or a component of the vector $\tilde{\mathbf{b}}$.

\subsubsection{The second fuzzy number is a non-diagonal element of the matrix $\tilde{A}$}

Let us assume that the second fuzzy number is a non-diagonal element of the matrix $\tilde{A}$. We first fix the second fuzzy number on the lower and the upper bound of its support. In that way we can find the solutions for the lower and upper line of the Figure 1 . We also fix the second fuzzy number on its modal value. Thereafter the parametric functions with the second fuzzy number as variable and a fixed first fuzzy number are calculated by using the earlier obtained parametric functions. We first solve the following system of linear equations:

$$
\left\{\begin{array}{l}
f_{\text {denom }}^{l}\left(F n_{1}^{*}\right)=\underline{F n_{2}}{ }^{2} c_{4}^{*}+\underline{F n_{2}} c_{5}^{*}+c_{6}^{*} \\
f_{\text {denom }}^{m}\left(F n_{1}^{*}\right)=\left({\widetilde{F n_{2}}}_{1}^{2} c_{4}^{*}+\left(\widetilde{F n}_{2}\right)_{1} c_{5}^{*}+c_{6}^{*}\right. \\
f_{\text {denom }}^{u}\left(F n_{1}^{*}\right)={\overline{F n_{2}}}^{2} c_{4}^{*}+\overline{F n_{2}} c_{5}^{*}+c_{6}^{*} ;
\end{array}\right.
$$

where $f_{\text {denom }}^{l}\left(F n_{1}^{*}\right), f_{\text {denom }}^{m}\left(F n_{1}^{*}\right)$ and $f_{\text {denom }}^{l}\left(F n_{1}^{*}\right)$ are the denominators of the parametric functions for respectively the lower $(l)$ bound of the support, the modal value $(m)$ and the upper $(u)$ bound of the support of $\widetilde{F n_{2}}$, with the first fuzzy number as variable and evaluated in the fixed value $F n_{1}^{*}$. Thereafter we calculate the parameters $c_{1 j}^{*}, c_{2 j}^{*}$ and $c_{3 j}^{*}$ by solving the following system:

$$
\left\{\begin{array}{l}
f_{n u m, j}^{l}\left(F n_{1}^{*}\right)=\underline{F n}^{2} c_{1 j}^{*}+\underline{F n} c_{2 j}^{*}+c_{3 j}^{*} \\
f_{n u m, j}^{m}\left(F n_{1}^{*}\right)=(\widetilde{F n})_{1}^{2} c_{1 j}^{*}+(\widetilde{F n})_{1} c_{2 j}^{*}+c_{3 j}^{*} \\
f_{\text {num }, j}^{u}\left(F n_{1}^{*}\right)=\overline{F n}^{2} c_{1 j}^{*}+\overline{F n} c_{2 j}^{*}+c_{3 j}^{*}
\end{array}\right.
$$

where $f_{\text {num }, j}^{l}\left(F n_{1}^{*}\right), f_{\text {num }, j}^{m}\left(F n_{1}^{*}\right)$ and $f_{\text {num }, j}^{l}\left(F n_{1}^{*}\right)$ are the numerators of the $j$-th component of the parametric functions for respectively the lower $(l)$ bound of the support, the modal value $(m)$ and the upper $(u)$ bound of the support of $\widetilde{F n_{2}}$, with the first fuzzy number as variable and evaluated in the 
fixed value $F n_{1}^{*}$. The function $f_{j}^{*}$ is then given by, for all $j \in\{1, \ldots, n\}$,

$$
f_{j}^{*}\left(F n_{2}\right)=\frac{\left(F n_{2}\right)^{2} c_{1 j}^{*}+F n_{2} c_{2 j}^{*}+c_{3 j}^{*}}{\left(F n_{2}\right)^{2} c_{4}^{*}+F n_{2} c_{5}^{*}+c_{6}^{*}},
$$

for all $F n_{2} \in \operatorname{supp}\left(\widetilde{F n_{2}}\right)$.

\subsubsection{The second fuzzy number is a component of the vector $\mathbf{b}$}

When the second fuzzy number is located in the right-hand side of the system of linear fuzzy equations, i.e. , when we have for instance that $\widetilde{\mathbf{b}}=$ $\left(\tilde{b}_{1}, b_{2}, \ldots, b_{n}\right)$, one sees immediately that $c_{3}^{*}=0$ and $c_{4}^{*}=|A|=f_{\text {denom }}^{l}\left(F n_{1}^{*}\right)=f_{\text {denom }}^{u}\left(F n_{1}^{*}\right)$. Then we have to calculate the values for the parameters $c_{1 j}^{*}$ and $c_{2 j}^{*}$ by using the numerators of the parametric functions for the first fuzzy coefficient:

$$
\left\{\begin{array}{l}
f_{\text {num }, j}^{l}\left(F n_{1}^{*}\right)=\underline{F n_{2}} c_{1 j}^{*}+c_{2 j}^{*} \\
f_{\text {num }, j}^{u}\left(F n_{1}^{*}\right)=\overline{F n_{2}} c_{1 j}^{*}+c_{2 j}^{*}
\end{array}\right.
$$

for $j \in\{1, \ldots, n\}$. The function $f_{j}^{*}$ is then given by, for all $j \in\{1, \ldots, n\}$,

$$
f_{j}^{*}\left(F n_{2}\right)=\frac{F n_{2} c_{1 j}^{*}+c_{2 j}^{*}}{f_{\text {denom }}^{l}\left(F n_{1}^{*}\right)},
$$

for all $F n_{2} \in \operatorname{supp}\left(\widetilde{F n_{2}}\right)$.

The solution $\tilde{x}_{j}^{\text {sym }}$ is obtained similarly as in (12) in all the three cases (Subsection 3.2.1, 3.2.2 and 3.2.3).

\subsection{Systems with more than two fuzzy coefficients}

Clearly, the procedure proposed in Subsection 3.2 can be extended to systems with more than two fuzzy coefficients. In Figure 2 the method is illustrated for 3 fuzzy coefficients. First we calculate all the parametric functions and solutions for the front and the back face of the cube. Thereafter we obtain the parametric functions and solutions corresponding to the lines between the front and the back face of the cube by using the parameters obtained earlier.

Example 1. Consider the following system of linear fuzzy equations:

$$
\tilde{A} \tilde{\mathbf{x}}=\tilde{\mathbf{b}}
$$

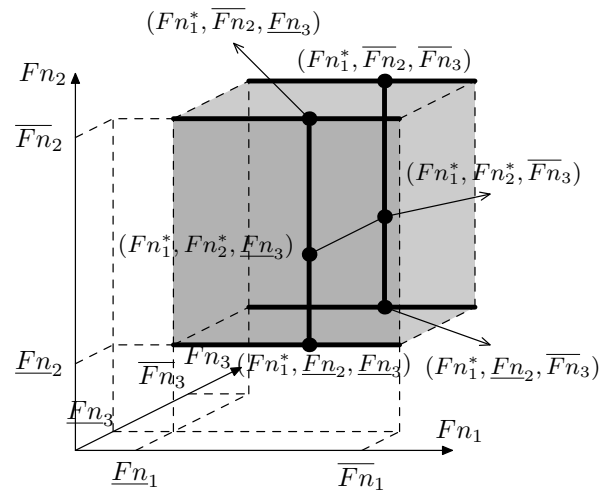

Fig. 2: Solving systems with three fuzzy coefficients.
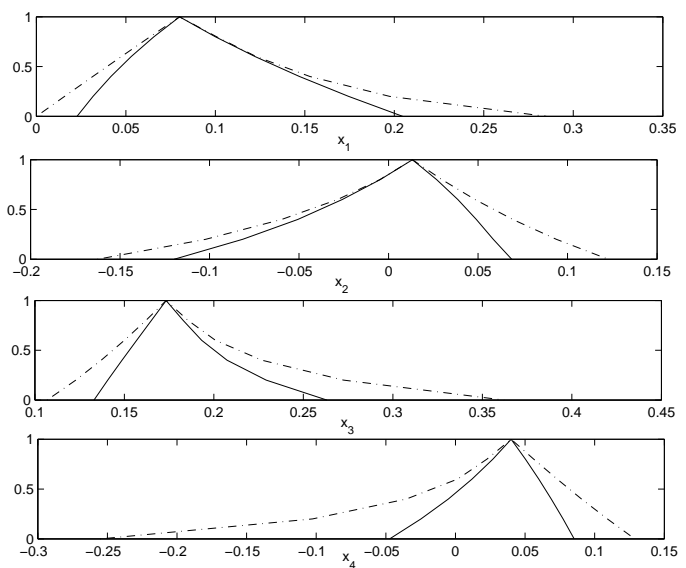

Fig. 3: Solutions for the system of Example 1 with the symmetry of the matrix $\tilde{A}$ taken into account (full line) and without taking the symmetry into account (interrupted line).

where $\tilde{A}=$

$$
\begin{aligned}
& \left(\begin{array}{cccc}
1 & (3 / 4 / 5) & (4 / 5 / 6) & 0 \\
(3 / 4 / 5) & -4 & (1 / 4 / 6) & (0 / 1 / 3) \\
(4 / 5 / 6) & (1 / 4 / 6) & 2 & 5 \\
0 & (0 / 1 / 3) & 5 & 3
\end{array}\right) \\
& \tilde{\mathbf{b}}=\left(\begin{array}{l}
1 \\
1 \\
1 \\
1
\end{array}\right)
\end{aligned}
$$

This system has a symmetric matrix $\tilde{A}$. The solution for this system is obtained on the one hand without taking the symmetry into account and on the other hand taking the symmetry into account. The difference in solution for both approaches is shown in Figure 3. When the symmetry is not taken into account, the solution is an overestimation. 


\section{Conclusion}

In this paper we have proposed a method to solve $n \times n$ systems in which some (or all) coefficients are fuzzy and in which we take the symmetry of the matrix $\tilde{A}$ into account. While in the method of Buckley and $\mathrm{Qu}$ for every element in the support of each fuzzy number the corresponding crisp $n \times n$ system is solved, in our method only the crisp $n \times n$ systems corresponding to the bounds of each support and the modal value of each fuzzy number must be solved, and the other necessary solutions for the combinations of the lower and the upper bounds of the considered $\alpha$-level are obtained by evaluating parametric functions. By considering the symmetry of the matrix $\tilde{A}$, the obtained solution is a better solution, there is no overestimation. As an interesting side-effect we find that the computation time is considerably reduced.

\section{Acknowledgment}

Annelies Vroman would like to thank the Fund for Scientific Research-Flanders for funding the research project G.0476.04 elaborated on in this paper.

\section{References}

[1] J. J. Buckley and Y. Qu, Solving systems of linear fuzzy equations. Fuzzy Sets and Systems, 43: 33-43, Elsevier, 1991.

[2] R. Moore, Interval Arithmetic, Prentice-Hall, Englewood Cliffs, NJ, USA, 1966.

[3] E. E. Kerre, Fuzzy Sets and Approximate Reasoning, Xian Jiaotong University Press, Xian, People's Republic of China, 1999.

[4] A. Vroman, G. Deschrijver and E. E. Kerre, Solving systems of linear fuzzy equations by parametric functions. IEEE Transactions on Fuzzy Systems, in press.

[5] A. Vroman, G. Deschrijver and E. E. Kerre, Solving systems of linear fuzzy equations by parametric functions - An improved algorithm. Fuzzy Sets and Systems, 158:1515-1534, 2007. 